\title{
Engineering Tobacco Mosaic Virus, Barley Stripe Mosaic Virus, and their Virus-Like-Particles for Synthesis of Biotemplated Nanomaterials
}

\author{
Kok Zhi Lee ${ }^{1}$, Vindula Basnayake Pussepitiya ${ }^{2}$, Yu-Hsuan Lee ${ }^{1}$, Sue Loesch-Fries ${ }^{1}$, Michael \\ Harris $^{1}$, Shohreh Hemmati ${ }^{2}$, and Kevin Solomon ${ }^{1}$ \\ ${ }^{1}$ Purdue University \\ ${ }^{2}$ Oklahoma State University Stillwater
}

July 7,2020

\begin{abstract}
Biomolecules are increasingly attractive templates for the synthesis of functional nanomaterials. Chief among them are the plant Tobacco mosaic virus (TMV) and Barley stripe mosaic virus (BSMV) due to their high aspect ratio, narrow size distribution, diverse biochemical functionalities presented on the surface, and compatibility with a number of chemical conjugations. These properties are also easily manipulated by genetic modification to enable the synthesis of a range of metallic and non-metallic nanomaterials for diverse applications. This article reviews the characteristics of TMV, BSMV, and their virus-like particle (VLP) derivatives and how these may be manipulated to extend their use and function. A focus of recent efforts has been on greater understanding and control of the self-assembly processes that drive biotemplate formation. We briefly outline how these features have been exploited in engineering applications such as sensing, catalysis, and energy storage, and discuss emerging advances that promise to accelerate the development of these biotemplates for widescale industrial use.
\end{abstract}

\section{Introduction}

Nanoscale materials offer precise tuning of material properties through atomistic control of matter and energy interactions at increasingly small length scales. This precision in material properties enables vast new opportunities in sensing, data storage, energy storage, and catalysis, among other areas [1-5]. Key to this process is the synthesis of nanoscale materials with well-defined and uniform architectures $[4,6]$. Traditional chemical and physical synthesis technologies rely on purely material- and energy-intensive processes that are difficult to control and scale, rely on toxic or otherwise non-green chemicals, have non-uniform outputs, and limited control of atomistic features [7-10]. In contrast, biology synthesizes uniform nanoscale biomolecules via well-defined design rules, which may be engineered and serve as biotemplates for the synthesis of metallic nanomaterials [11, 12]. Biomolecules, such as nucleic acids, microtubules, amyloid fibers, and viruses, have been used as scaffolds for the construction of hierarchical complex nanomaterials [13-17]. Their surfaces present diverse biochemical functionalities that are used to organize nanoparticle synthesis, and may be modified via conjugation with organic or inorganic materials to create novel devices and control metal mineralization [18, 19]. Finally, biomolecules possess well-defined nanoscale architectures, are structurally stable across a wide range of conditions, and can be easily manipulated via genetic engineering. All these features make biomolecules attractive biotemplates for bottom-up nanomaterial assembly.

Viruses possess many advantages over other types of biomolecules for nanoparticle synthesis as they occur in a wide range of shapes and sizes, and present diverse chemical functionalities for synthesis. Plant viruses are widely used because they are harmless to human beings [11]. For instance, Cowpea chlorotic mottle virus, Cowpea mosaic virus, and Brome mosaic virus form icosahedral structures that range in size from 
18-30 nm while Tobacco mosaic virus and Barley stripe mosaic virus assume rod-shaped structures up to $300 \mathrm{~nm}$ in length [11]. This diversity enables biotemplating of diverse nanomaterials for incorporation as catalysts, sensors, battery anodes, and semiconductor digital memory devices [1-5]. Viral particles consist of self-assembled capsid proteins (CPs) and nucleic acids that genetically encode the CPs. The CPs present diverse biochemical functionalities via amino acid residues on the particle surface that interact with metals in solution and drive nanoparticle synthesis. These residues may be conjugated to other compounds to enable synthesis of different nanomaterials and create novel functional properties [18, 19]. Similarly, the presented protein functionalities and dimensions can be directly modified via engineering the encoding nucleic acid sequence without dramatically altering viral structure to enable synthesis of new materials $[1,20,21]$.

Non-infectious virus-like particles (VLPs) may be generated via heterologous expression of CPs in non-native species without using the complete viral genome [22]. Expressed CPs spontaneously self-assemble into VLPs that possess the same rich chemical diversity on their surfaces to drive nanoparticle synthesis. Plant VLPs also offer several compelling features over real viruses for VLP engineering and industrial-scale production. First, VLPs are more tolerant of mutations than live virus enabling more engineering opportunities to enhance function. For example, genetic modifications that enhance particle structural stability to improve nanomaterial synthesis yields enable the formation of nucleic acid-free VLPs that are unable to infect host cells [23]. VLP production does not rely on infection for production and may be stably produced with this enhancement in a heterologous host. Second, heterologous microbial hosts replicate and produce CPs much more rapidly than plants, which need several weeks to grow and mature before infection with virus for production [24]. Moreover, live viruses are infectious agents and must be grown in a Biosafety Level 2 greenhouse by plant virologists to contain potential environmental contamination, adding to their costs [25]. Third, bacterial VLP production also leverages a wealth of bioprocessing infrastructure that has been developed for large scale production of food, pharmaceuticals, and chemicals [26]. Thus, VLPs are more compelling platforms for the development of viral biotemplates.

Tobacco mosaic virus (TMV) is widely used for biotemplating due to its architecture and physicochemical properties (Table 1). The dimensions of TMV are well suited to biotemplating applications such as the production of batteries and sensors $[1,6,27]$. TMV is self-assembled from over a thousand copies of a single $\mathrm{CP}$ into a $300 \mathrm{~nm}$ long nanotube whose inner and outer diameters are $4 \mathrm{~nm}$ and $18 \mathrm{~nm}$, respectively [28]. This aspect ratio maximizes the available surface area in compact volumes enabling more efficient battery electrodes with higher charge densities, and increased sensitivity to chemical analytes as sensors. Moreover, the biochemical/physicochemical properties of TMV enable reduction of metal ions and nanoparticle synthesis on the template under ambient conditions [3, 29]. Finally, TMV and its VLPs consist of a single CP that is amenable to genetic and chemical modifications that expand the types of nanomaterials that may be synthesized, enhance morphological uniformity, and increase particle density [30]. While TMV is the most commonly used in bionanotechnology, the evolutionarily-related Barley stripe mosaic virus (BSMV) provides a promising alternative biotemplate [16]. BSMV has similar architecture to TMV (Table 1) but presents distinct surface functional groups that accelerate nanoparticle synthesis and increase nanoparticle density for increased electrical and thermal conductivities and analyte sensitivity as sensors [16]. Moreover, the evolutionary similarities between TMV and BSMV may allow successful engineering strategies from TMV to be applied in BSMV to expand and enhance the properties of BSMV-derived biotemplates. Thus, BSMV is emerging as an attractive virus biotemplate for nanomaterial synthesis.

The convergence of advances from materials science, structural biology, molecular biology, chemistry, machine learning, engineering, and synthetic biology, now enable the rapid engineering and development of TMV, BSMV, and their VLPs for biotemplating. Their physicochemical properties make them well-suited for the synthesis of diverse nanomaterials for applications such as catalysis, energy storage, and sensing. In this review, we provide an overview of the properties and use of TMV, BSMV and their VLPs for nanoparticle synthesis, and focus on emerging technologies and approaches to engineer VLPs to enhance their function and broaden the nanomaterials that may be synthesized.

\section{Viral particle self-assembly and metal nanoparticle synthesis}


Viral biotemplates such as TMV and BSMV spontaneously self-assemble from identical CP subunits due to several covalent, hydrogen, and electrostatic interactions encoded within the CP primary sequence and its associated nucleic acids (Figure 1) [34]. These interactions must be strong enough to withstand the pHs, temperatures, and ionic strengths required for successful nanoparticle synthesis. CP is first translated and folded before ultimately forming flat disks via hydrophobic interactions between residues on the CP surface [34]. An RNA sequence naturally found in the TMV viral genome, known as the origin of assembly sequence or OAS, assumes a hairpin secondary structure that then serves as a nucleus for nanotube formation. CP disks are threaded by OAS-containing RNA, allowing for electrostatic interactions between the OAS and $\mathrm{CP}$ [35]. This interaction forces the $\mathrm{CP}$ disk to assume a helical conformation, reorienting themselves by induced proton adsorption and subsequent hydrogen-bonding, which then rapidly polymerizes with other CP molecules into a nanotube [34]. Strengthening the final assembly are electrostatic interactions between adjacent CPs, which are mediated by a handful of negatively charged residues in a motif known as the Caspar carboxylate cluster [36]. Calcium ions typically neutralize the repulsive negative charges of adjacent CPs and drive viral polymerization. Control of these interactions via genetic engineering of the $\mathrm{CP}$ and/or the OAS offers tremendous potential to modify nanotube architecture and its stability in subsequent biotemplating $[23,37]$.

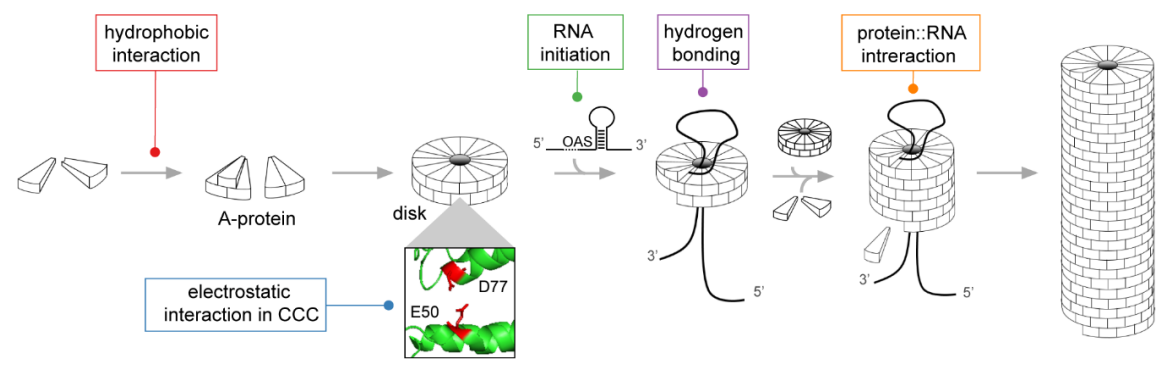

Figure 1. Driving forces for TMV assembly. TMV CP experiences various interactions, including hydrophobic interactions, RNA initiation, electrostatic interactions in the Caspar carboxylate cluster (CCC), hydrogen bonding, and RNA-protein interaction at different stages of assembly.

Metal nanoparticles are frequently synthesized spontaneously on viral biotemplates using aqueous metal solutions. The metal precursor ions adsorb and are chemically reduced on the viral particle surfaces at many adsorption and nucleation sites to form a metallic nanomaterial $[38,39]$. The chemical interactions that drive metal precursor adsorption and reduction are not well understood. However, the adsorption process is frequently described by a single-step Langmuir isotherm that is solely driven by covalent interactions, e.g. palladium on TMV [38]. As metal ion precursor adsorption and reduction are the fundamental processes that drives metal coating formation, the oxidation potential of surface accessible residues must be sufficient to drive metal reduction (Table 2). Amino acid residues that are easily oxidized, such as cysteine, tyrosine, and lysine, more readily interact with metals driving deposition [40-42]. Metals with higher positive reduction potentials, including gold, silver, and platinum, can be reduced by the various functional groups present on the CP of TMV (Table 2) [39]. This deposition is frequently enhanced by engineering the amino acids residues that are presented on the virus/VLP [30,39]. Other metals such as nickel, iron, and cobalt cannot be reduced this way as they have negative reduction potentials. Instead, a different metal that is more readily reduced such as palladium is mineralized first onto the $\mathrm{CP}$, which then serves as a catalyst to reduce target metal ions to metal atoms [43,44]. For example, nickel and cobalt are deposited in the inner channel of TMV after mineralization of TMV with Pd and Pt [44]. Fundamentally, appropriate pairing of amino acid side chain that can chemically reduce and interact with metals intrinsically sets the metal adsorption capacity and controls the rate of reaction as nanoparticle synthesis proceeds spontaneously under ambient conditions.

Plant-produced BSMV has been demonstrated to be a viable biotemplate for mineralization of palladium nanowires, however, biomineralization with BSMV differs from that of TMV [16]. The surface of BSMV 
allows metal ion precursor deposition to proceed via a multi-step Langmuir isotherm that incorporates both electrostatic and covalent adsorbent-adsorbate interactions. This difference may arise in part due to the larger amount of BSMV surface-exposed residues, compared to TMV, in an unstructured insertion loop containing 10 amino acids that protrudes from the particle surface [33]. These stronger interactions increase the adsorption capacity for Pd on BSMV two-fold compared to that on TMV [16]. Similarly, the rate of adsorption is increased compared to TMV, suggesting that BSMV can be fully coated in fewer processing cycles saving both time and expensive precursor material. Furthermore, BSMV biotemplates produce more uniformly sized nanoparticles relative to TMV. The additional opportunities to engineer metal deposition via the insertion loop and superior adsorption and metal nanoparticle synthesis characteristics make BSMV an attractive alternative to TMV that may generate more uniform metal nanostructures more economically.

\section{Nanostructure Synthesis with Viral Biotemplates for Diverse Applications}

Nanostructures are increasingly sought to create advanced materials that improve electrical conductivity and capacitance, current generation, catalytic activity, and detection sensitivity [3, 5, 19, 46, 47]. These applications benefit from high aspect ratio nanomaterials that can be biotemplated on TMV, BSMV or their VLPs. However, each application relies on distinct metals whose unique electronic structure is key to enabling these applications; e.g. metal with high conductivity such as copper is used in conductive nanowires while gold is relatively inert making it an ideal substrate for a sensor [19, 48]. Moreover, the precise function of the nanomaterial in these roles relies on their incorporation into devices with unique architectures (Figure 2 ). To enable the synthesis of these nanomaterials, the viral biotemplates are modified and processed in innovative ways; we describe only a handful below. 

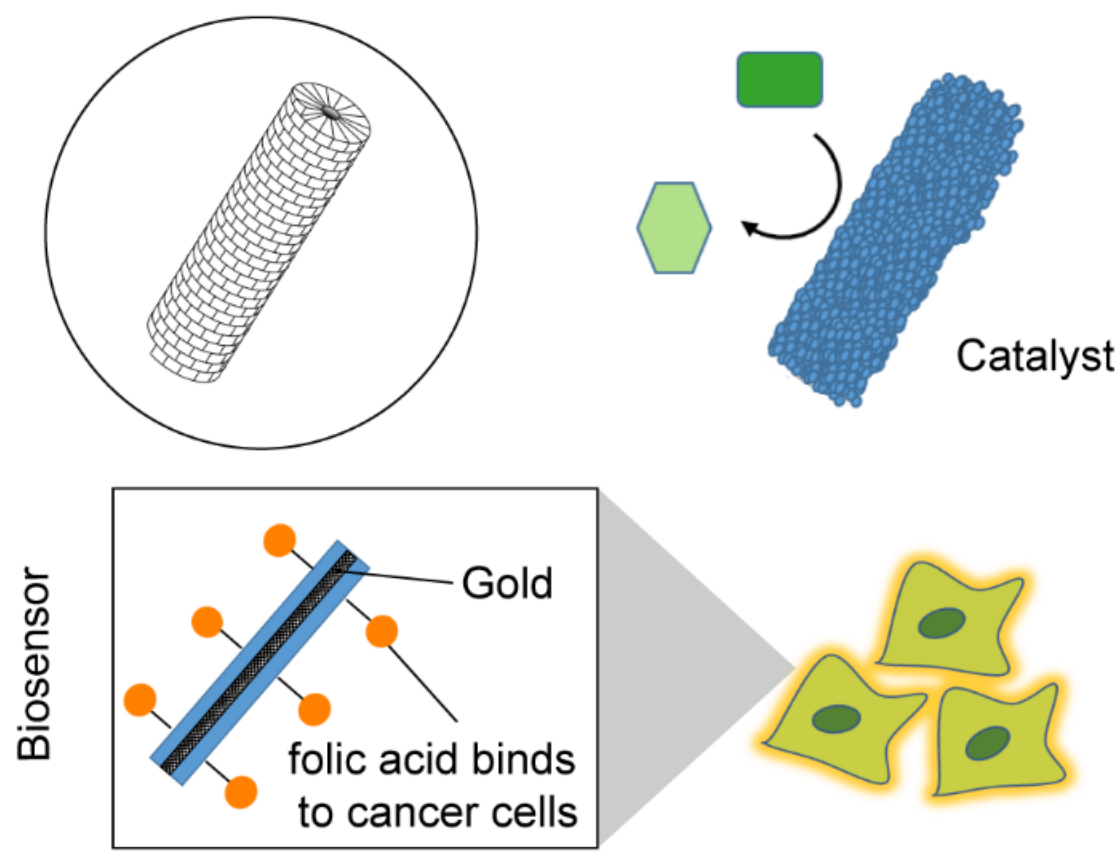

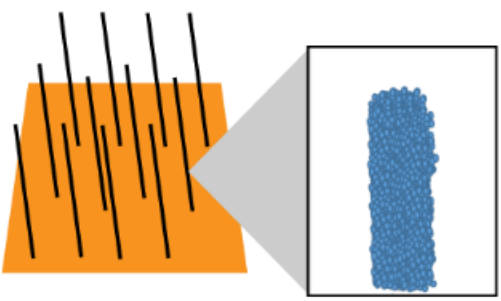

Electrode in battery

\section{Ruthenium oxide coated TMV}

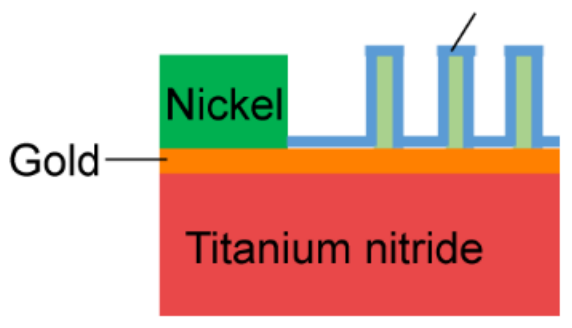

Super microcapacitor

Figure 2 . Some potential applications of nanomaterials templated on TMV, BSMV and their VLPs.

\subsection{Metal Nanowires}

Metal nanowires are used for a multitude of applications from electronics and sensors to lasers due to their improved conductivity [49]. Industrial-scale production of metal nanowires is most commonly achieved via polyol synthesis. However, it is not environmentally-friendly or sustainable as it uses various toxic polyhydric reducing agents such as ethylene glycol and propylene glycol in an energy-intensive reaction process that occurs at high temperatures [8, 50-52]. In contrast, highly conductive and uniform nanowires may be synthesized without toxic reagents or high temperatures via templating on TMV or its VLP [53]. To enable creation of palladium, platinum or gold nanowires, additional lysine, cysteine, and/or tyrosine side chains are added to the surface of the viral template to provide sufficient reducing power for nanoparticle synthesis and potential nucleation sites for metal binding. The introduction of this reduction power can decrease or completely remove the need for additional reducing agents in nanowire synthesis, thereby lowering costs $[29$, $54]$.

For thinner nanowires, metal may be deposited in the narrow viral channel of TMV, rather than on the surface $[55,56]$. Buffering agents are used to alter the ionic state of amino acid side chains, changing its 
electrostatic interactions to decrease metal deposition on the surface. The inner channel, whose side chains are distinct from the surface, remain charged and electrostatically interact with metal solutions to adsorb and reduce synthesized metal nanoparticles. These techniques may be directly applied to platinum or palladium nanowires; however, nanowires composed of other metals such as copper, cobalt, and/or nickel require a core of $\mathrm{Pd}$ and $\mathrm{Pt}$, which then serves as catalytic sites for reduction of other metals forming thin alloy nanowires such as $\mathrm{CoFe}, \mathrm{CoNi}, \mathrm{FeNi}$, and $\mathrm{CoFeNi}[44,55]$. Bimetallic ferromagnetic alloys with $\mathrm{Pt}$ such as $\mathrm{CoPt}$ and $\mathrm{FePt}_{3}$ may also be synthesized directly by mixing precursor metal salt solutions with platinum or palladium salts in the same deposition bath thus simplifying the two-step process [43].

\subsection{Batteries and Energy Storage}

Batteries produce current via chemical reactions on the surface of electrodes, which is limited by the available surface area [3]. To generate the currents needed to power modern electronic devices, metal coated TMV virions can be vertically oriented on top of a gold substrate to form a carpet-like nanoforest structure (Figure 2 ). The vertical attachment is achieved by introducing a cysteine residue at the amino terminus of CP to produce TMV1cys virions [3,6]. Most cysteines in TMV are partially recessed and thus are incapable of metal binding; however, the N-terminal residue is fully exposed allowing for near-vertical assembly of the biotemplate on a gold substrate via covalent interactions between the gold and the thiol group of the cysteine. Subsequent coating with nickel and/or cobalt increases the active electrode surface area more than ten-fold with a doubling in electrode discharge capacity (or current generation) [3]. Similarly, Pd/Ni/Si-coated TMV anodes in lithium ion batteries can increase the discharge capacity by nearly ten-fold compared to then available graphite anodes [6]. Finally, incorporating TMV in sodium-ion batteries as a carbon/tin/nickelcoated TMV anode can increase battery cycling lifespan with little degradation in charge capacity over 150 deep charging cycles. This capability made it the longest-cycling nano-Sn anode material for Na-ion batteries at the time [27]. Coated TMV patterned with a similar nanoforest structure has also been shown to increase the performance of micro supercapacitors (Figure 2), which also rely on surface area to store charges [57].

\subsection{Catalysis}

Catalyst effectiveness is proportional to its exposed surface area, as more sites are available for reaction. To minimize the costs of large volumes of catalytic metal, an economical strategy for catalyst synthesis is to deposit catalytic metal on an inexpensive inert substrate with a high surface area to volume ratio such as carbon or a biotemplate. However, biotemplated catalysts are more efficient; Pd catalyst synthesized from TMV1cys immobilized on a gold substrate in a nanostar configuration increased the reaction rate $68 \%$ relative to commercial $\mathrm{Pd} / \mathrm{C}$ catalyst of comparable size [58]. TMV-templated catalysts are stable and can be recycled with negligible degradation in performance over several cycles $[4,5,58]$. The superior performance of TMV-templated catalyst is attributed primarily to two factors. One, palladium particles mineralized on TMV tend to be more uniform as compared to commercially available palladium catalyst supported on carbon material leading to more efficient catalysis [58, 59]. And secondly, fabrication of palladium on TMV does not use surfactants and capping agents like the synthesis of traditional catalysts that can block active sites [60]. Removal of these surfactants to rescue catalysis is incomplete and adds to the cost of catalyst manufacture. Thus, biotemplated catalysts are both more catalytically- and economically- advantaged in many scenarios.

\subsection{Sensors}

Sensors are used in a wide range of areas including environmental and disease monitoring to detect and quantify selected analytes from complex dirty solutions. Effective sensors must have high selectivity, sensitivity, and structural stability over the range of environmental conditions they are likely to experience. Selectivity for a specific analyte in mixed samples is achieved by functionalizing the viral surface with metals, enzymes and/or other chemicals. For example, palladium-coated TMV rapidly and reversibly detects hydrogen at room temperatures [47]. Similarly, gold coated nanowires conjugated with folic acid via coupling agents can interact with the folate receptors on tumor cells to detect cancers with high sensitivity [19]. Additionally, TMV may be decorated with biotin to enable binding of enzymes to detect penicillin [61]. Other desirable 
sensor properties such as sensitivity and stability arise from the nature of viral biotemplates themselves. TMV's high surface area to volume ratio allows for more contact with analytes, amplifying signal generation and improving device sensitivity [62]. Similarly, TMV structure is very stable in high temperatures and over a wide range of pHs (Table 1) that may be encountered in the analysis of complex mixtures, making biosensors derived from TMV more durable and rugged.

\section{Engineering Viruses and VLPs for Enhanced Biotemplate Properties}

Improved understanding of TMV and BSMV structural biology and molecular biology has informed a number of strategies to control and enhance biotemplate properties. These include improved structural stability to enable synthesis of more diverse nanomaterials, control of VLP architecture and enhanced uniform coating to control physical properties, and more versatile conjugation capabilities to tune function. These modifications are powered by the flexibility of the $\mathrm{CP}$-encoding nucleic acids, which can be readily engineered via genetic tools.

\subsection{Structural stability in high temperatures and extreme $\mathrm{pH}$}

Despite the successes in coating TMV, BSMV and their VLPs with metals such as gold, palladium and platinum, many industrially-desirable metals are not readily deposited due to unfavorable electrochemistries (Table 2) [30,63]. This issue may be mitigated somewhat through the use of buffering and reducing agents, activation by primary deposition with another metal such as $\mathrm{Pt}$, and higher temperatures to create more favorable processing conditions. These strategies ultimately increase metal-template interactions via altering residue ionization state, changing the reduction potentials of the metal ions and amino acid side chains, and/or create nucleation sites to drive metal mineralization. However, these conditions may destabilize the CP interactions that drive self-assembly of the template leading to biotemplate loss and low yields of metal mineralization. Biotemplates are only stable within fixed $\mathrm{pH}$ and temperature ranges (Table 1). To address this challenge, TMV and VLPs have been engineered to increase their structural stability to resist disassembly.

The stability of TMV biotemplates has been increased by control of the intermolecular forces that drive selfassembly (Figure 1), enabling more rapid biotemplating of a wider range of materials [64-66]. For example, single point mutations within the Caspar carboxylate cluster can enhance viral assembly. Neutralization of a negatively-charged residue (E50Q) or replacing a negatively-charged residue with a positively-charged residue in the Caspar carboxylate center (E50R or D77R) has been shown to produce longer virions that spontaneously self-assemble without an RNA that contain an OAS or other nucleic acids in transgenic plants [67]. In similar fashion, neutralizing the negative-charged residues with site-specific mutations, E50Q/D77N, was sufficient to rescue TMV-VLP assembly in E.coli even in the absence of RNA with an OAS, which typically results in viral disks alone [21]. In combination with cysteine engineering, E50Q/D77N mutants are able to attach to a gold-coated plate [21]. These mutations within the Caspar carboxylate cluster are sufficient to overcome the poor stability of nucleic acid-free VLPs and recover resistance to $\mathrm{pH}$, attaining similar stability to wildtype virions. In so doing, they create nucleic acid-free VLPs that have a free internal channel for synthesis of thin nanowires [64]. Engineering of the hydrophobic interactions between CPs (Figure 1) may further improve the stability of VLPs; however, modifications of this nature have yet to be evaluated.

OAS-containing nucleic acids that initiate the assembly of wildtype virus and VLPs also act as a molecular ruler that sets the length of produced VLPs [20]. While VLPs that have been engineered appropriately (e.g. at the Caspar carboxylate cluster) can self-assemble without this molecular ruler, the resulting VLPs are more heterogenous in size than OAS-containing viral particles with sizes as small as $20 \mathrm{~nm}$ [23]. Thus, future work should include efforts to control the precise dimensions of VLPs or development of efficient separation technologies to isolate VLPs with specific dimensions.

\subsection{Coating uniformity}

Nanomaterial properties are controlled in part by the coating uniformity or smoothness of the surface of metal 
deposited on biotemplate. This uniformity relies on the molecular interactions between the deposited metal and residues on the surface or in the channel of the biotemplate. Coating agents such as citric acid are added to the deposition reaction to alter the charge of surface-exposed residues and increase metal-biotemplate interactions during the metal deposition process [39, 68, 69]. However, these $\mathrm{pH}$-sensitive electrostatic interactions can be disrupted by other reagents of the deposition process leading to poor nanoparticle synthesis and nonuniform coating.

To address this challenge, covalent bonds that are resistant to modest $\mathrm{pH}$ changes can be introduced to drive nanoparticle synthesis. Site specific mutagenesis of the capsid protein to introduce cysteine and lysine residues has been demonstrated to improve nanoparticle synthesis [30, 70]. These residues may be introduced either on the surface or inner channel of TMV VLPs (e.g. S3C, T103C, T158K) or on either terminus of the $\mathrm{CP}$ [70-72]. These modifications allow for metal deposition under a wider range of $\mathrm{pHs}$ and promote high density nucleation sites for nanoparticle synthesis, unlike wildtype whose nanoparticle synthesis is driven by weaker surface drying effects. For example, two cysteine residues have been genetically added to the amino-terminus of TMV CP, resulting in 4260 available thiol groups per VLP for metal reduction and deposition [30]. The introduced thiol groups on the TMV surface serve as a reaction site for nanomaterials such as gold and palladium to reduce metal ions or form covalent bonds with non-metal materials via thiol coupling. Cysteine enhances the deposition of several metals including gold, silver, and palladium, and also enables novel attachments for device manufacture (e.g. nanoforests for battery anode synthesis) [30]. Lysine substitution has also served as a functional group for chemical conjugation of silica coatings [70].

\subsection{Diversifying the nanomaterials that can be synthesized on TMV/BSMV-derived biotem- plates}

While TMV/BSMV virions and VLPs serve as exceptional biotemplates for metal mineralization, they are not directly compatible for the synthesis of non-metallic nanomaterials. However, the introduction of surfaceaccessible amino acids, including aspartate, glutamate, and tyrosine provide conjugation sites for synthesis of non-metallic nanomaterials via covalent bonding. For example, the carboxylic acid moiety of aspartate and glutamate can react with amines, which have been functionalized with biotin, chromophores, and crown ethers while tyrosine can conjugate with PEG [73]. These conjugations enable medical applications of TMV such as vectors for drug delivery.

In addition to canonical amino acids, synthetic noncanonical amino acids with unique functional groups have also been incorporated in other VLPs such as hepatitis B virus (HBV) or bacteriophage Q $\beta$ to expand coating capabilities further [74]. Noncanonical amino acids provide unique functional groups, such as alkynes and aminophenylalanine, that facilitate bioorthogonal coating [75]. Bioorthogonal reactions enable specific conjugation via the noncanonical amino acids without cross-reaction with canonical amino acids of the virions/VLPs, reducing the risk of disrupting the physicochemical properties that drive biotemplate selfassembly. Alkyne-containing noncanonical amino acids have been incorporated into virions to attach PEG, oligonucleotides, antibody fragments, and other peptides via click chemistry [74] while aminophenylalanine incorporated VLPs have been used to conjugate cell type-specific targeting peptides, enabling drug delivery to specific cell types [76]. The number of novel viral-derived biomaterials are certain to expand further as new noncanonical amino acids are engineered and incorporated into CPs [77].

\section{Future directions}

Current advances in a number of fields now converge to enhance the function and potential of viral biotemplates. These activities are greatly accelerated by the innovations of synthetic biology that enable more rapid building and testing of engineered VLPs. These constructs are thoroughly characterized with spectroscopic techniques with the rich datasets fed to machine learning algorithms to guide iterative design and improvements (Figure 3). Several emerging opportunities are as follows. 


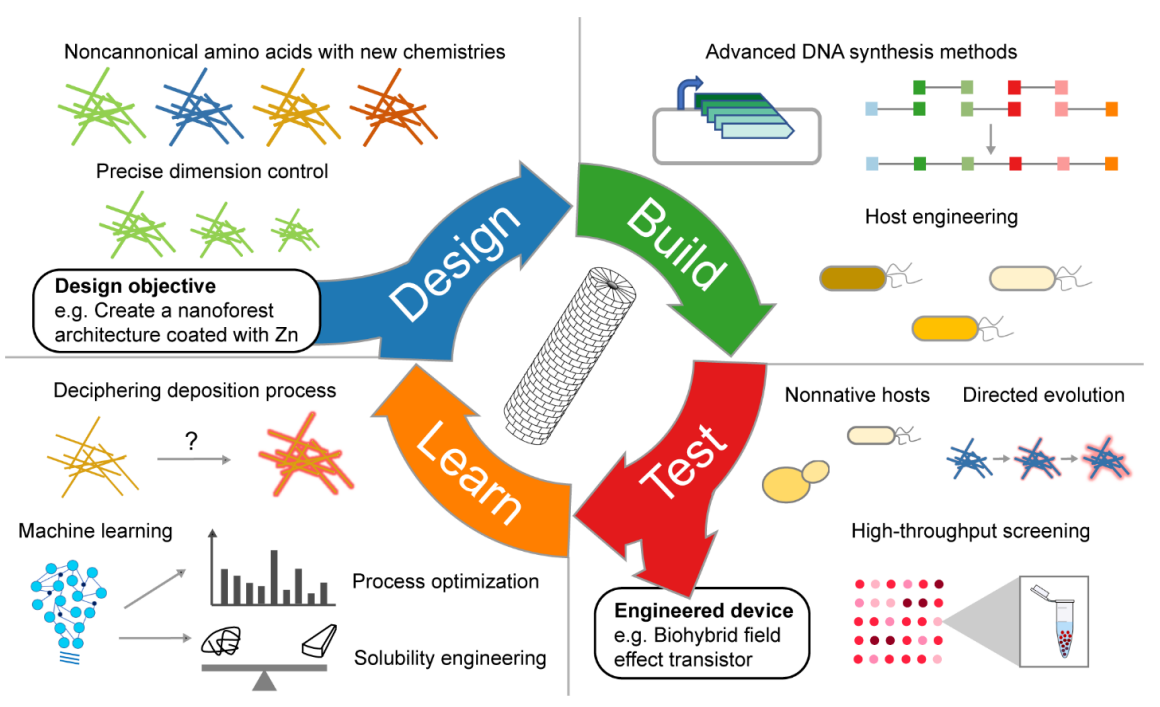

Figure 3. Emerging opportunities to accelerate development of TMV, BSMV and their VLPs. In the "design" phase, VLPs with specific properties (e.g. conjugation to an enzyme via noncanonical amino acids) for an application guide the design of DNA sequences that are then rapidly assembled in the "build" phase. Advanced DNA synthesis methods rapidly assemble DNA to produce thousands of variants while host engineering boosts VLP production. In the "test" phase, these constructs are expressed and screened. Directed evolution develops VLPs with desirable features while cell-free systems enable high-throughput screening. In the "learn" phase, characterization of the resulting products and analysis via empirical and machine learning approaches refine the initial design ultimately leading to an optimal biotemplate and bioproduction platform.

\subsection{Rapid prototyping and screening of novel biotemplates}

Despite the exciting future for VLP development, the production and characterization of newly engineered VLPs are time-consuming processes, spanning several days. Thus, new screening tools are necessary to expedite the development of newly engineered VLPs. Cell-free systems that contain the transcriptional and translational machinery needed for VLP production could become integral to rapid VLP prototyping and characterization without laborious purification. Cell-free systems can be used to produce engineered VLP candidates in a highly parallel or high-throughput manner with minimal inputs due to their microliter scale reaction volumes [78]. VLPs derived from non-plant viruses are already being developed via cellfree technologies. For example, a cell-free system was used to incorporate noncanonical amino acids in bacteriophage MS2 and bacteriophage Q $\beta$ for click-chemistry functionalization [79].

Rapid screening is also needed to identify engineered mutants with desirable properties. Advanced DNA synthesis methods such as Gibson and Golden-gate assemblies can produce thousands of DNA-encoding VLPs variants in a single reaction [80-82]. However, screening of these variants for improved function requires characterization of individual mutants, which makes the screening process slow and inefficient. Instead of screening every variant, directed evolution can be applied to evolve mutants with desirable traits [83]. Directed evolution is a process where mutants are propagated and those with desirable properties are selected for. The ability of a given mutant to propagate or replicate is linked to the property that is desired, allowing surviving mutants to 'select' for enhanced properties. Thus, thousands of variants can be simultaneously evaluated in hours without screening of every single variant. The selected variants can then be mutated to introduce additional genetic diversity and subject to another round of selection. The iteration of these processes can generate protein mutants with optimized or even new properties such as producing VLPs with enhanced structural stability [84]. Although directed evolution has not been widely used for VLP engineering, the recent development of new directed evolution methods, including assisted 
machine learning [85], which identifies more promising mutations to be constructed and screened, andin vivo continuous directed evolution [86], which streamlines the genetic engineering process, will accelerate the engineering of VLPs.

\subsection{Improved biotemplate production yields}

VLPs frequently form insoluble aggregates when expressed in non-native hosts that are unsuitable for biotemplating processes [87]. To increase the yield of usable VLP biotemplates, the solubility of heterologous CP must be enhanced. There are many ways to increase protein solubility [88]; however, increasing CP solubility for VLPs production is challenging as one of the drivers of protein insolubility, strong hydrophobic interactions, is also the driving force for virion and VLP assembly. However, machine learning algorithms have begun to identify the design rules for soluble VLP CP design without disrupting VLP self-assembly based on hydrophobicity scales [89]. An early report with these tools counterintuitively suggests that CP solubility may be highly correlated with arginine content in some VLPs, despite its negative correlation with solubility of short chain variable fragments. Arginine reduces many hydrophobic protein-protein interactions while interacting favorably with other amino acids to reduce random aggregation. However, these tools have yet to be applied to forward engineer novel VLP CPs.

\subsection{Molecular understanding and control of the mineralization process}

While the basic processes of metal mineralization are qualitatively understood, mechanistic details regarding the reaction mechanisms of metal reduction and adsorption remain elusive. Current studies on metal mineralization using TMV, BSMV and their corresponding VLPs as biotemplates focused on characterization of synthesized nanostructures using different techniques such as transmission electron microscopy (TEM) [24], Fourier transform infrared spectroscopy (FTIR), and X-ray scattering analysis [19, 59]. These methods reveal the structure of the final synthesized structure and inform hypotheses of how metal mineralization has occurred. However, a more effective approach to understand how metal mineralization takes place is to perform in-situ FTIR [90]. This allows direct observation of the reaction progress enabling determination of the mechanism of mineralization. It is also possible to observe how changes in reaction conditions such as $\mathrm{pH}$, temperature, and concentrations of precursors, reducing agents and biotemplate would affect metal mineralization including particle size, particle size homogeneity, and the type of metal nanostructures mineralized on the surface. This would require an extensive design of experiments to systematically evaluate the effect of each parameter and their interactions. Machine learning algorithms such as artificial neural networks have already been applied to these rich datasets to create predictive models for nanoparticle synthesis as a function of processing parameters [91]. Similarly, neural networks first used to predict the binding of metallic ion cofactors to enzymes could be extended to VLPs to predict and model metal-biotemplate interactions as a function of engineered CP protein sequence [92]. Such computational tools would greatly accelerate biotemplate engineering efforts and optimize deposition processes for metallic nanomaterial synthesis.

\section{Concluding remarks}

Integrated concepts from material science, biology, chemistry, and engineering have developed TMV, BSMV, and their VLPs for the synthesis of high-quality nanomaterials for diverse applications. TMV and BSMV are particularly attractive as biotemplates due to their ease of manipulation, large scale production in bacterial culture, and surface moieties that facilitate interactions with a number of materials. New insights afforded by structural biology and spectroscopic characterization now inform the engineering of CP molecular interactions within a viral particle/VLP, and between the viral particle/VLP and deposited nanomaterials. These have led to a number of innovative genetic strategies that enhance biotemplate structural stability and improve the uniformity and properties of synthesized nanoscale structures enabling diverse applications such as sensing, catalysis, and energy storage. While further engineering is needed to scale up these systems for widespread industrial nanomaterial synthesis, emerging technologies from synthetic biology and machine learning promise to accelerate the pace of TMV- and BSMV- derived biotemplate development in the years to come.

\section{Acknowledgement}


This review was partially supported by the USDA National Institute of Food and Agriculture (Hatch Multistate Project S1075) and the Robert B and Virginia V. Covalt Endowed Professorship of Chemical Engineering at Purdue. KZL is partially supported by Purdue Research Foundation Fellowships (\#60000025 \& \#60000029) and a Ralph W. and Grace M. Showalter Research Trust Award (\#41000622) to KVS. VBP is supported by startup funds from the College of Engineering, Architecture and Technology at Oklahoma State University. YHL is supported by the Robert B and Virginia V. Covalt Endowed Professorship of Chemical Engineering awarded to MTH. The funding sources had no role in the study design, data collection, data analysis, decision to publish, or preparation of the manuscript.

\section{Conflict of interest}

The authors declare no financial or commercial conflict of interest.

\section{References:}

[1] Koch, C., Wabbel, K., Eber, F.J., Krolla-Sidenstein, P., et al., Modified TMV Particles as Beneficial Scaffolds to Present Sensor Enzymes. Front. Plant Sci. 2015, 6 , DOI: 10.3389/fpls.2015.01137.

[2] Portney, N.G., Tseng, R.J., Destito, G., Strable, E., et al., Microscale memory characteristics of virusquantum dot hybrids.Appl. Phys. Lett. 2007, 90 , 214104.

[3] Royston, E., Ghosh, A., Kofinas, P., Harris, M.T., et al., Self-Assembly of Virus-Structured High Surface Area Nanomaterials and Their Application as Battery Electrodes. Langmuir 2008,24 , 906-912.

[4] Yang, C., Manocchi, A.K., Lee, B., Yi, H., Viral templated palladium nanocatalysts for dichromate reduction. Applied Catalysis B: Environmental 2010, 93 , 282-291.

[5] Yang, C., Manocchi, A.K., Lee, B., Yi, H., Viral-templated palladium nanocatalysts for Suzuki coupling reaction. J. Mater. Chem. 2010, 21, 187-194.

[6] Chen, X., Gerasopoulos, K., Guo, J., Brown, A., et al., Virus-Enabled Silicon Anode for Lithium-Ion Batteries. ACS Nano2010, 4 , 5366-5372.

[7] Mueller, R., Mädler, L., Pratsinis, S.E., Nanoparticle synthesis at high production rates by flame spray pyrolysis. Chemical Engineering Science 2003, 58 , 1969-1976.

[8] Sun, Y., Xia, Y., Large-Scale Synthesis of Uniform Silver Nanowires Through a Soft, Self-Seeding, Polyol Process. Advanced Materials 2002, 14 , 833-837.

[9] Cattaneo, S., Althahban, S., Freakley, S.J., Sankar, M., et al., Synthesis of highly uniform and compositioncontrolled gold-palladium supported nanoparticles in continuous flow. Nanoscale 2019,11 , 8247-8259.

[10] Khorsand Zak, A., Majid, W.H.Abd., Ebrahimizadeh Abrishami, M., Yousefi, R., et al., Synthesis, magnetic properties and X-ray analysis of Zn0.97X0.03O nanoparticles ( $\mathrm{X}=\mathrm{Mn}, \mathrm{Ni}$, and $\mathrm{Co}$ ) using Scherrer and size-strain plot methods. Solid State Sciences 2012, 14 , 488-494.

[11] Zhang, Y., Dong, Y., Zhou, J., Li, X., et al., Application of Plant Viruses as a Biotemplate for Nanomaterial Fabrication. Molecules 2018, 23 , 2311.

[12] Lee, S.-Y., Lim, J.-S., Harris, M.T., Synthesis and application of virus-based hybrid nanomaterials. Biotechnology and Bioengineering 2012, 109 , 16-30.

[13] Watson, S.M.D., Mohamed, H.D.A., Horrocks, B.R., Houlton, A., Electrically conductive magnetic nanowires using an electrochemical DNA-templating route. Nanoscale 2013, 5 , 5349-5359.

[14] Zhou, J.C., Gao, Y., Martinez-Molares, A.A., Jing, X., et al., Microtubule-Based Gold Nanowires and Nanowire Arrays. Small 2008,4, 1507-1515.

[15] Malisauskas, M., Meskys, R., Morozova-Roche, L.A., Ultrathin silver nanowires produced by amyloid biotemplating. Biotechnology Progress 2008, 24 , 1166-1170. 
[16] Adigun, O.O., Retzlaff-Roberts, E.L., Novikova, G., Wang, L., et al., BSMV as a Biotemplate for Palladium Nanomaterial Synthesis.Langmuir 2017, 33 , 1716-1724.

[17] Chu, S., Brown, A.D., Culver, J.N., Ghodssi, R., Tobacco Mosaic Virus as a Versatile Platform for Molecular Assembly and Device Fabrication. Biotechnology Journal 2018, 13 , 1800147.

[18] Brown, A.D., Naves, L., Wang, X., Ghodssi, R., et al., Carboxylate-Directed In Vivo Assembly of Viruslike Nanorods and Tubes for the Display of Functional Peptides and Residues.Biomacromolecules 2013, 14 , 3123-3129.

[19] Qu, Y., Yang, Y., Du, R., Zhao, M., Peroxidase activities of gold nanowires synthesized by TMV as template and their application in detection of cancer cells. Appl Microbiol Biotechnol 2020,104 , 3947-3957.

[20] Saunders, K., Lomonossoff, G.P., In Planta Synthesis of Designer-Length Tobacco Mosaic VirusBased Nano-Rods That Can Be Used to Fabricate Nano-Wires. Front Plant Sci 2017, 8 , DOI: 10.3389/fpls.2017.01335.

[21] Brown, A.D., Naves, L., Wang, X., Ghodssi, R., et al., Carboxylate-Directed In Vivo Assembly of Viruslike Nanorods and Tubes for the Display of Functional Peptides and Residues.Biomacromolecules 2013, 14 , 3123-3129.

[22] Zeltins, A., Construction and Characterization of Virus-Like Particles: A Review. Mol Biotechnol 2013, $53,92-107$.

[23] Kadri, A., Wege, C., Jeske, H., In vivo self-assembly of TMV-like particles in yeast and bacteria for nanotechnological applications. Journal of Virological Methods 2013, 189 , 328-340.

[24] Jeong, H., Seong, B.L., Exploiting virus-like particles as innovative vaccines against emerging viral infections. J Microbiol. 2017, $55,220-230$.

[25] Makarov, V.V., Skurat, E.V., Semenyuk, P.I., Abashkin, D.A., et al., Structural Lability of Barley Stripe Mosaic Virus Virions.PLoS One 2013, 8 , DOI: 10.1371/journal.pone.0060942.

[26] Schugerl, K., Hubbuch, J., Integrated bioprocesses.Current Opinion in Microbiology 2005, 8 , 294-300.

[27] Liu, Y., Xu, Y., Zhu, Y., Culver, J.N., et al., Tin-Coated Viral Nanoforests as Sodium-Ion Battery Anodes. ACS Nano 2013,7, 3627-3634.

[28] Alonso, J.M., Gorzny, M.Ł., Bittner, A.M., The physics of tobacco mosaic virus and virus-based devices in biotechnology. Trends in Biotechnology 2013, 31 , 530-538.

[29] Freer, A.S., Guarnaccio, L., Wafford, K., Smith, J., et al., SAXS characterization of genetically engineered tobacco mosaic virus nanorods coated with palladium in the absence of external reducing agents. Journal of Colloid and Interface Science 2013,392 , 213-218.

[30] Lee, S.-Y., Royston, E., Culver, J.N., Harris, M.T., Improved metal cluster deposition on a genetically engineered tobacco mosaic virus template. Nanotechnology 2005, 16 , S435-S441.

[31] Oster, G., The Isoelectric Points of Some Strains of Tobacco Mosaic Virus. J. Biol. Chem. 1951, 190 , $55-59$.

[32] King, A.M.Q., Adams, M.J., Carstens, E.B., Lefkowitz, E.J. (Eds.), Family - Virgaviridae, in: Virus Taxonomy, Elsevier, San Diego 2012, pp. 1139-1162.

[33] Clare, D.K., Pechnikova, E.V., Skurat, E.V., Makarov, V.V., et al., Novel Inter-Subunit Contacts in Barley Stripe Mosaic Virus Revealed by Cryo-Electron Microscopy. Structure 2015, 23 , 1815-1826.

[34] Kegel, W.K., van der Schoot, P., Physical Regulation of the Self-Assembly of Tobacco Mosaic Virus Coat Protein. Biophysical Journal 2006, 91 , 1501-1512. 
[35] Butler, P.J., Self-assembly of tobacco mosaic virus: the role of an intermediate aggregate in generating both specificity and speed.Philos Trans R Soc Lond B Biol Sci 1999, 354 , 537-550.

[36] Wang, H., Planchart, A., Stubbs, G., Caspar Carboxylates: The Structural Basis of Tobamovirus Disassembly. Biophysical Journal1998, 74, 633-638.

[37] Wege, C., Koch, C., From stars to stripes: RNA-directed shaping of plant viral protein templatesstructural synthetic virology for smart biohybrid nanostructures. WIREs Nanomedicine and Nanobiotechnology 2020, 12 , e1591.

[38] Adigun, O.O., Novikova, G., Retzlaff-Roberts, E.L., Kim, B., et al., Decoupling and elucidation of surface-driven processes during inorganic mineralization on virus templates. Journal of Colloid and Interface Science 2016, 483 , 165-176.

[39] Dujardin, E., Peet, C., Stubbs, G., Culver, J.N., et al., Organization of Metallic Nanoparticles Using Tobacco Mosaic Virus Templates. Nano Lett. 2003, 3 , 413-417.

[40] Bechtel, T.J., Weerapana, E., From structure to redox: the diverse functional roles of disulfides and implications in disease.Proteomics 2017, 17 .

[41] Aeschbach, R., Amadoò, R., Neukom, H., Formation of dityrosine cross-links in proteins by oxidation of tyrosine residues.Biochimica et Biophysica Acta (BBA) - Protein Structure 1976,439 , 292-301.

[42] Utrera, M., Rodríguez-Carpena, J.-G., Morcuende, D., Estévez, M., Formation of Lysine-Derived Oxidation Products and Loss of Tryptophan during Processing of Porcine Patties with Added Avocado Byproducts. J. Agric. Food Chem. 2012, 60 , 3917-3926.

[43] Tsukamoto, R., Muraoka, M., Seki, M., Tabata, H., et al., Synthesis of CoPt and FePt3 Nanowires Using the Central Channel of Tobacco Mosaic Virus as a Biotemplate. Chem. Mater. 2007,19 , 2389-2391.

[44] Knez, M., Bittner, A.M., Boes, F., Wege, C., et al., Biotemplate Synthesis of 3-nm Nickel and Cobalt Nanowires. Nano Lett. 2003, 3 , 1079-1082.

[45] Bratsch, S.G., Standard Electrode Potentials and Temperature Coefficients in Water at 298.15 K. Journal of Physical and Chemical Reference Data 1989, 18 , 1-21.

[46] Freer, A.S., Gilpin, C., Mueller, L., Harris, M., A Novel Method to Determine the Resistance of Biotemplated Nanowires. Chemical Engineering Communications 2015, 202 , 1216-1220.

[47] Srinivasan, K., Cular, S., Bhethanabotla, V.R., Sang Yup Lee, et al., Nanomaterial sensing layer based surface acoustic wave hydrogen sensors, in: IEEE Ultrasonics Symposium, 2005. , 2005, pp. 645-648.

[48] Zhou, J.C., Soto, C.M., Chen, M.-S., Bruckman, M.A., et al., Biotemplating rod-like viruses for the synthesis of copper nanorods and nanowires. Journal of Nanobiotechnology 2012, 10 , 18.

[49] Bergin, S.M., Chen, Y.-H., Rathmell, A.R., Charbonneau, P., et al., The effect of nanowire length and diameter on the properties of transparent, conducting nanowire films. Nanoscale 2012, 4 , 1996-2004.

[50] Choi, Y.-H., Chae, Y.-S., Lee, J.-H., Kwon, Y., et al., Mechanism of metal nanowire formation via the polyol process.Electron. Mater. Lett. 2015, 11 , 735-740.

[51] Hemmati, S., Barkey, D.P., Eggleston, L., Zukas, B., et al., Silver Nanowire Synthesis in a Continuous Millifluidic Reactor.ECS J. Solid State Sci. Technol. 2017, 6 , P144.

[52] Hemmati, S., Barkey, D.P., Gupta, N., Banfield, R., Synthesis and Characterization of Silver Nanowire Suspensions for Printable Conductive Media. ECS J. Solid State Sci. Technol. 2015,4, P3075.

[53] Wnek, M., Górzny, M.Ł., Ward, M.B., Wälti, C., et al., Fabrication and characterization of gold nanowires templated on virus-like arrays of tobacco mosaic virus coat proteins. Nanotechnology 2013, 24, 025605. 
[54] Lim, J.-S., Kim, S.-M., Lee, S.-Y., Stach, E.A., et al., Biotemplated Aqueous-Phase Palladium Crystallization in the Absence of External Reducing Agents. Nano Lett. 2010, 10 , 3863-3867.

[55] Balci, S., Hahn, K., Kopold, P., Kadri, A., et al., Electroless synthesis of $3 \mathrm{~nm}$ wide alloy nanowires insideTobacco mosaic virus.Nanotechnology 2012, 23, 045603.

[56] Balci, S., Bittner, A.M., Hahn, K., Scheu, C., et al., Copper nanowires within the central channel of tobacco mosaic virus particles.Electrochimica Acta 2006, 51, 6251-6257.

[57] Gnerlich, M., Ben-Yoav, H., Culver, J.N., Ketchum, D.R., et al., Selective deposition of nanostructured ruthenium oxide using Tobacco mosaic virus for micro-supercapacitors in solid Nafion electrolyte. Journal of Power Sources 2015, 293 , 649-656.

[58] Yang, C., Meldon, J.H., Lee, B., Yi, H., Investigation on the catalytic reduction kinetics of hexavalent chromium by viral-templated palladium nanocatalysts. Catalysis Today 2014, 233 , 108-116.

[59] Manocchi, A.K., Seifert, S., Lee, B., Yi, H., In Situ Small-Angle X-ray Scattering Analysis of Palladium Nanoparticle Growth on Tobacco Mosaic Virus Nanotemplates. Langmuir 2011, 27, 7052-7058.

[60] Li, D., Wang, C., Tripkovic, D., Sun, S., et al., Surfactant Removal for Colloidal Nanoparticles from Solution Synthesis: The Effect on Catalytic Performance. ACS Catal. 2012, 2 , 1358-1362.

[61] Koch, C., Poghossian, A., Schöning, M.J., Wege, C., Penicillin Detection by Tobacco Mosaic Virus -Assisted Colorimetric Biosensors. Nanotheranostics 2018, 2 , 184-196.

[62] Zhou, H., Liu, J., Xu, J.-J., Zhang, S., et al., Chapter Two - Advances in DNA/RNA detection using nanotechnology, in: Makowski, G.S. (Ed.), Advances in Clinical Chemistry, 1st ed., Elsevier, Cambridge, MA 2019, pp. 31-98.

[63] Lee, S.-Y., Choi, J., Royston, E., Janes, D.B., et al., Deposition of Platinum Clusters on Surface-Modified Tobacco Mosaic Virus. Journal of Nanoscience and Nanotechnology 2006, 6 , 974-981.

[64] Kadri, A., Maiß, E., Amsharov, N., Bittner, A.M., et al., Engineered Tobacco mosaic virus mutants with distinct physical characteristics in planta and enhanced metallization properties. Virus Research 2011, 157 , $35-46$.

[65] Royston, E., Lee, S.-Y., Culver, J.N., Harris, M.T., Characterization of silica-coated tobacco mosaic virus. Journal of Colloid and Interface Science 2006, 298 , 706-712.

[66] Atanasova, P., Hoffmann, R.C., Stitz, N., Sanctis, S., et al., Engineered nanostructured virus/ZnO hybrid materials with dedicated functional properties. Bioinspired, Biomimetic and Nanobiomaterials 2019, $8,2-15$.

[67] Bendahmane, M., Chen, I., Asurmendi, S., Bazzini, A.A., et al., Coat protein-mediated resistance to TMV infection of Nicotiana tabacum involves multiple modes of interference by coat protein. Virology2007, $366,107-116$.

[68] Khan, A.A., Fox, E.K., Górzny, M.Ł., Nikulina, E., et al., pH Control of the Electrostatic Binding of Gold and Iron Oxide Nanoparticles to Tobacco Mosaic Virus. Langmuir 2013, 29 , 2094-2098.

[69] Knez, M., Sumser, M., Bittner, A.M., Wege, C., et al., Spatially Selective Nucleation of Metal Clusters on the Tobacco Mosaic Virus. Advanced Functional Materials 2004, 14, 116-124.

[70] Altintoprak, K., Seidenstücker, A., Welle, A., Eiben, S., et al., Peptide-equipped tobacco mosaic virus templates for selective and controllable biomineral deposition. Beilstein Journal of Nanotechnology 2015, 6 , 1399-1412.

[71] Geiger, F.C., Eber, F.J., Eiben, S., Mueller, A., et al., TMV nanorods with programmed longitudinal domains of differently addressable coat proteins. Nanoscale 2013, 5, 3808-3816. 
[72] Zhou, K., Li, F., Dai, G., Meng, C., et al., Disulfide Bond: Dramatically Enhanced Assembly Capability and Structural Stability of Tobacco Mosaic Virus Nanorods. Biomacromolecules 2013, 14 , 2593-2600.

[73] Schlick, T.L., Ding, Z., Kovacs, E.W., Francis, M.B., Dual-Surface Modification of the Tobacco Mosaic Virus. J. Am. Chem. Soc. 2005, 127, 3718-3723.

[74] Strable, E., Prasuhn, D.E., Udit, A.K., Brown, S., et al., Unnatural Amino Acid Incorporation into Virus-Like Particles.Bioconjugate Chem. 2008, 19 , 866-875.

[75] Boyce, M., Bertozzi, C.R., Bringing chemistry to life.Nature Methods 2011, 8, 638-642.

[76] Carrico, Z.M., Romanini, D.W., Mehl, R.A., Francis, M.B., Oxidative coupling of peptides to a virus capsid containing unnatural amino acids. Chem. Commun. 2008, 1205-1207.

[77] Dunkelmann, D.L., Willis, J.C.W., Beattie, A.T., Chin, J.W., Engineered triply orthogonal pyrrolysyltRNA synthetase/tRNA pairs enable the genetic encoding of three distinct non-canonical amino acids. Nature Chemistry 2020, 12, 535-544.

[78] Carlson, E.D., Gan, R., Hodgman, C.E., Jewett, M.C., Cell-Free Protein Synthesis: Applications Come of Age. Biotechnol Adv 2012,30 , 1185-1194.

[79] Patel, K.G., Swartz, J.R., Surface Functionalization of Virus-Like Particles by Direct Conjugation Using Azide-Alkyne Click Chemistry. Bioconjugate Chem. 2011, 22 , 376-387.

[80] Gibson, D.G., Young, L., Chuang, R.-Y., Venter, J.C., et al., Enzymatic assembly of DNA molecules up to several hundred kilobases. Nat Meth 2009, 6, 343-345.

[81] Engler, C., Kandzia, R., Marillonnet, S., A one pot, one step, precision cloning method with high throughput capability. PLoS ONE 2008, 3, e3647.

[82] Engler, C., Marillonnet, S., Combinatorial DNA assembly using Golden Gate cloning. Methods Mol. Biol. 2013, 1073 , 141-156.

[83] Packer, M.S., Liu, D.R., Methods for the directed evolution of proteins. Nat Rev Genet 2015, 16 , 379-394.

[84] Chapman, R., Bourn, W.R., Shephard, E., Stutz, H., et al., The Use of Directed Evolution to Create a Stable and Immunogenic Recombinant BCG Expressing a Modified HIV-1 Gag Antigen. PLOS ONE 2014,9 , e103314.

[85] Wu, Z., Kan, S.B.J., Lewis, R.D., Wittmann, B.J., et al., Machine learning-assisted directed protein evolution with combinatorial libraries. PNAS 2019, 116 , 8852-8858.

[86] Ravikumar, A., Arzumanyan, G.A., Obadi, M.K.A., Javanpour, A.A., et al., Scalable, Continuous Evolution of Genes at Mutation Rates above Genomic Error Thresholds. Cell 2018, 175 , 1946-1957.e13.

[87] Huo, Y., Wan, X., Ling, T., Wu, J., et al., Expression and purification of norovirus virus like particles in Escherichia coli and their immunogenicity in mice. Molecular Immunology 2018,93 , 278-284.

[88] Trevino, S.R., Scholtz, J.M., Pace, C.N., Measuring and Increasing Protein Solubility. Journal of Pharmaceutical Sciences2008, 97 , 4155-4166.

[89] Vormittag, P., Klamp, T., Hubbuch, J., Ensembles of Hydrophobicity Scales as Potent Classifiers for Chimeric Virus-Like Particle Solubility - An Amino Acid Sequence-Based Machine Learning Approach. Front. Bioeng. Biotechnol. 2020, 8 , DOI: 10.3389/fbioe.2020.00395.

[90] Ahn, D.J., Berman, A., Charych, D., Probing the Dynamics of Template-Directed Calcite Crystallization with in Situ FTIR. J. Phys. Chem. 1996, 100 , 12455-12461. 
[91] Shafaei, A., Khayati, G.R., A predictive model on size of silver nanoparticles prepared by green synthesis method using hybrid artificial neural network-particle swarm optimization algorithm.Measurement 2020, 151 , 107199.

[92] Naik, P.K., Ranjan, P., Kesari, P., Jain, S., MetalloPred: A tool for hierarchical prediction of metal ion binding proteins using cluster of neural networks and sequence derived features. JBPC2011, 02 , 112-123.

Table 1. Characteristics of TMV and BSMV viral particles

\begin{tabular}{lll}
\hline & TMV & BSMV \\
\hline Outer diameter $(\mathrm{nm})$ & $18^{\mathrm{a}}$ & $20^{\mathrm{c}}$ \\
Inner diameter $(\mathrm{nm})$ & $4^{\mathrm{a}}$ & $3-4^{\mathrm{d}}$ \\
Length (nm) & $300^{\mathrm{a}}$ & $110-150^{\mathrm{c}}$ \\
Thermal stability* () & $90^{\mathrm{a}}$ & $70^{\mathrm{c}}$ \\
pH stability & $3.0-9.0^{\mathrm{a}}$ & - \\
CP size $(\mathrm{kDa})$ & $17.6^{\mathrm{a}}$ & $22^{\mathrm{c}}$ \\
Copies Of CP & $2130^{\mathrm{a}}$ & $\sim 1385^{+}$ \\
isoelectric point $(\mathrm{pH})$ & $3.9^{\mathrm{b}}$ & $4.5^{\mathrm{c}}$ \\
\hline
\end{tabular}

*Limit at which virus is no longer infectious.

a [28]; ${ }^{\mathrm{b}}[31] ;{ }^{\mathrm{c}}[32] ;{ }^{\mathrm{d}}[33] ;{ }^{+}$estimated from helix pitch and CPs per turn [25]

- not measured

Table 2. Reduction potential of some metals at 25 C[45].

\begin{tabular}{lll}
\hline Species & Half-Reaction & Reduction Potential (V) \\
\hline $\mathbf{A u}$ & $\mathrm{Au}^{3+}+3 \mathrm{e}^{-}=\mathrm{Au}_{(\mathrm{s})}$ & 1.52 \\
$\mathbf{P t}$ & $\mathrm{Pt}^{2}++2 \mathrm{e}^{-}=\mathrm{Pt}_{(\mathrm{s})}$ & 1.20 \\
$\mathbf{P d}$ & $\mathrm{Pd}^{2+}+2 \mathrm{e}^{-}=\mathrm{Pd}_{(\mathrm{s})}$ & 0.92 \\
$\mathbf{A g}$ & $\mathrm{Ag}^{+}+\mathrm{e}^{-}=\mathrm{Ag}_{(\mathrm{s})}$ & 0.80 \\
$\mathbf{C u}$ & $\mathrm{Cu}^{+}+\mathrm{e}^{-}=\mathrm{Cu}_{(\mathrm{s})}$ & 0.52 \\
$\mathbf{N i}$ & $\mathrm{Ni}^{2+}+2 \mathrm{e}^{-}=\mathrm{Ni}_{(\mathrm{s})}$ & -0.24 \\
$\mathbf{F e}$ & $\mathrm{Fe}^{2+}+2 \mathrm{e}^{-}=\mathrm{Fe}_{(\mathrm{s})}$ & -0.44 \\
$\mathbf{C o}$ & $\mathrm{Co}^{2+}+2 \mathrm{e}^{-}=\mathrm{Co}_{(\mathrm{s})}$ & -0.28 \\
$\mathbf{F e}$ & $\mathrm{Fe}^{3+}+3 \mathrm{e}^{-}=\mathrm{Fe}_{(\mathrm{s})}$ & -0.04 \\
\hline
\end{tabular}

\section{Figure Legends}

Figure 1. Driving forces for TMV assembly. TMV CP experiences various interactions, including hydrophobic interactions, RNA initiation, electrostatic interactions in the Caspar carboxylate cluster (CCC), hydrogen bonding, and RNA-protein interaction at different stages of assembly.

Figure 2. Some potential applications of nanomaterials templated on TMV, BSMV and their VLPs.

Figure 3. Emerging opportunities to accelerate development of TMV, BSMV and their VLPs. In the "design" phase, VLPs with specific properties (e.g. conjugation to an enzyme via noncanonical amino acids) for an application guide the design of DNA sequences that are then rapidly assembled in the "build" phase. Advanced DNA synthesis methods rapidly assemble DNA to produce thousands of variants while host engineering boosts VLP production. In the "test" phase, these constructs are expressed and screened. 
Directed evolution develops VLPs with desirable features while cell-free systems enable high-throughput screening. In the "learn" phase, characterization of the resulting products and analysis via empirical and machine learning approaches refine the initial design ultimately leading to an optimal biotemplate and bioproduction platform. 